\title{
Improved actions and lattice coarsening effects in MCRG studies in SU(2) LGT
}

\author{
E.T. Tomboulis* and Alexander Velytsky ${ }^{\dagger}$ \\ Department of Physics and Astronomy, UCLA, Los Angeles, CA 90095-1547, USA \\ E-mail: tombouli@physics.ucla.edu, velephysics.ucla.edu
}

We study decimation procedures and effective (improved) actions in the framework of Monte Carlo Renormalization Group (MCRG). Particular attention is paid to matching the form of the effective action to the decimation procedure parameters. Using the static quark-antiquark potential in SU(2) LGT we probe different distance scales and find that an effective action containing multiple group representations is capable of reproducing long distance physics well. In particular, appropriate matching results in the practical elimination of the coarsening/fining effect of the lattice spacing under decimation. The short distance regime of the effective theory is also studied. We next carry out studies of effective actions involving both multiple representations and loops beyond the single plaquette towards determining an improved action good over a wide length scale regime.

The XXV International Symposium on Lattice Field Theory

July 30 - August 42007

Regensburg, Germany

\footnotetext{
* Speaker.

${ }^{\dagger}$ Current address: EFI, University of Chicago, 5640 S. Ellis Ave., Chicago, IL 60637 and Argonne National Laboratory, 9700 Cass Ave., Argonne, IL 60439
} 


\section{Decimations and Observables}

We report on our ongoing study of decimation procedures and effective actions in the framework of MCRG. It is based on our previous work [1, 2, 的 and constitutes a natural continuation. The aim is to extend the effective actions studied in these previous works so as to obtain a good representation of the physics of the original (undecimated) theory over a wide range - from short distance (rotational invariance) to long distances (string tension).

We employ two decimation procedures doubling the lattice spacing at various values of parameter $c$ (relative staple weight):

- Swendsen decimation

$$
Q_{\mu}(n)=U_{\mu}(n) U_{\mu}(n+\hat{\mu})+c \sum_{v \neq \mu} U_{v}(n) U_{\mu}(n+\hat{v}) U_{\mu}(n+\hat{v}+\hat{\mu}) U_{-v}(n+\hat{v}+2 \hat{\mu})
$$

- Double Smeared Blocking (DSB)

$$
\begin{aligned}
& U_{\mu}(n)=(1-6 c) U_{\mu}(n)+c \sum_{v \neq \mu} U_{v}(n) U_{\mu}(n+\hat{v}) U_{v}^{\dagger}(n+\hat{\mu}) \quad \times 2 \text { times } \\
& Q_{\mu}(n)=U_{\mu}(n) U_{\mu}(n+\hat{\mu}) .
\end{aligned}
$$

First we look at a single plaquette multi-representation effective action:

$$
S=\sum_{j=1 / 2}^{N_{r}} \beta_{j}\left[1-\frac{1}{d_{j}} \chi_{j}\left(U_{p}\right)\right] .
$$

The demon method is used for measurements of couplings. For more details of the simulations we refer to the mentioned references.

We compare observables (at different length scales) on the decimated and effective-actiongenerated configurations

$$
\frac{\Delta W_{N \times N}}{W_{N \times N}^{d e c}}=\frac{W_{N \times N}^{g e n}-W_{N \times N}^{d e c}}{W_{N \times N}^{d e c}} .
$$

Results are shown in Table 11. It is important to notice that demon measurements performed at the length scale of a plaquette do not preserve plaquette expectations ("no pinning"). In fact, as seen from Table [1, some decimation parameters tend to reproduce better long distance physics at the price of distorting short distance observables.

One should indeed note here that there is pronounced dependence on the value of the decimation parameter $c$. In particular, different trends as a function of scale are seen for different $c$ values.

Thus, the ability of a given chosen effective action to reproduce the original physics in a certain regime can vary substantially with 'tuning' of the decimation transformation parameters. We refer to this as matching the decimation prescription to the assumed form of the effective action.

At arbitrary $c$ value there is generally demon energy flow (Fig. 11), which is a sign of an on-going thermalization process during the microcanonical evolution. This is due to the fact that in general the decimated configurations are not equilibrium configurations of the effective action. Note that the value of $c$ with no demon flow is around the value giving better long distance behavior. 


\begin{tabular}{|c||c|c|c|c|}
\hline$c$ & $\beta_{1 / 2}, \beta_{1}, \beta_{3 / 2}, \ldots$ & $\Delta W_{2 \times 2} / W_{2 \times 2}^{\text {dec }}$ & $\Delta W_{3 \times 3} / W_{3 \times 3}^{d e c}$ & $\Delta W_{4 \times 4} / W_{4 \times 4}^{d e c}$ \\
\hline \hline 0.050 & $2.3536(5),-0.4208(9)$ & & & \\
& $0.1430(11),-0.0558(13)$ & $-0.1817(6)$ & $-0.637(1)$ & \\
& $0.0238(13),-0.0094(15)$ & & & \\
\hline 0.060 & $2.4660(7),-0.3635(11)$ & & & \\
& $0.1242(17),-0.0475(21)$ & $0.0105(7)$ & $-0.239(2)$ & \\
& $0.0195(25),-0.0070(24)$ & & & \\
\hline 0.063 & $2.4891(7),-0.3331(11)$ & & & \\
& $0.1140(14),-0.0436(19)$ & $0.0800(9)$ & $-0.049(3)$ & \\
& $0.0180(25),-0.0070(25)$ & & & \\
\hline 0.065 & $2.5023(7),-0.3098(12)$ & & & \\
& $0.1057(16),-0.0397(16)$ & $0.1305(9)$ & $0.106(3)$ & \\
& $0.0145(14),-0.0029(15)$ & & & \multirow{2}{*}{$0.290(19)$} \\
\hline 0.067 & $2.5125(7),-0.2832(16)$ & & & \\
& $0.0964(25),-0.0367(29)$ & $0.1774(9)$ & $0.266(3)$ & \\
& $0.0139(29)$ & & & \\
\hline 0.077 & $2.5463(11),-0.1167(17)$, & $0.4149(14)$ & $1.270(7)$ & \\
& $0.0320(23),-0.0055(28)$ & & & \\
\hline 0.1 & $2.4762(20), 0.4191(37)$ & & & \\
& $-0.1231(40), 0.0504(39)$ & $0.6558(9)$ & $2.627(6)$ & \\
& $-0.0191(53), 0.0063(54)$ & & & \\
\hline
\end{tabular}

Table 1: DSB decimations. Demon-measured couplings at different $c$ values, and difference of various size Wilson loops measured on decimated versus effective-action-generated configurations. Demon thermalization: 100 sweeps, measurements: 20 sweeps.

\section{Potential and string tension}

Next, we measure the string tension using the standard ansatz for the quark-antiquark potential. It is measured naively for the decimated configurations and using Lüscher-Weisz method for the effective-action-generated configurations. Having the string tension allows us to estimate any

\begin{tabular}{|c||c|c|}
\hline \multicolumn{1}{|c||}{} & \multicolumn{2}{c|}{$\sigma_{0}=0.0313(2)$} \\
\hline $\mathrm{c}$ & $\sigma_{\text {dec }}$ & $\sigma_{\text {gen }}$ \\
\hline 0.060 & $0.0271(37)$ & $0.0594(12)$ \\
0.065 & $0.0284(30)$ & $0.0385(14)$ \\
0.067 & $0.0291(49)$ & $0.0346(8)$ \\
0.068 & $0.0295(12)$ & $0.0292(9)$ \\
0.077 & $0.0285(24)$ & $0.0091(6)^{*}$ \\
\hline
\end{tabular}

Table 2: String tensions in original (undecimated) lattice units. $\sigma_{0}$ on $32^{3} \times 12, \sigma_{d e c}, \sigma_{\text {gen }}$ on $16^{3} \times 6$, except starred entry which is on $16^{3} \times 8$.

lattice spacing distortion beyond the decimation scaling factor (here equal to 2) resulting from the 


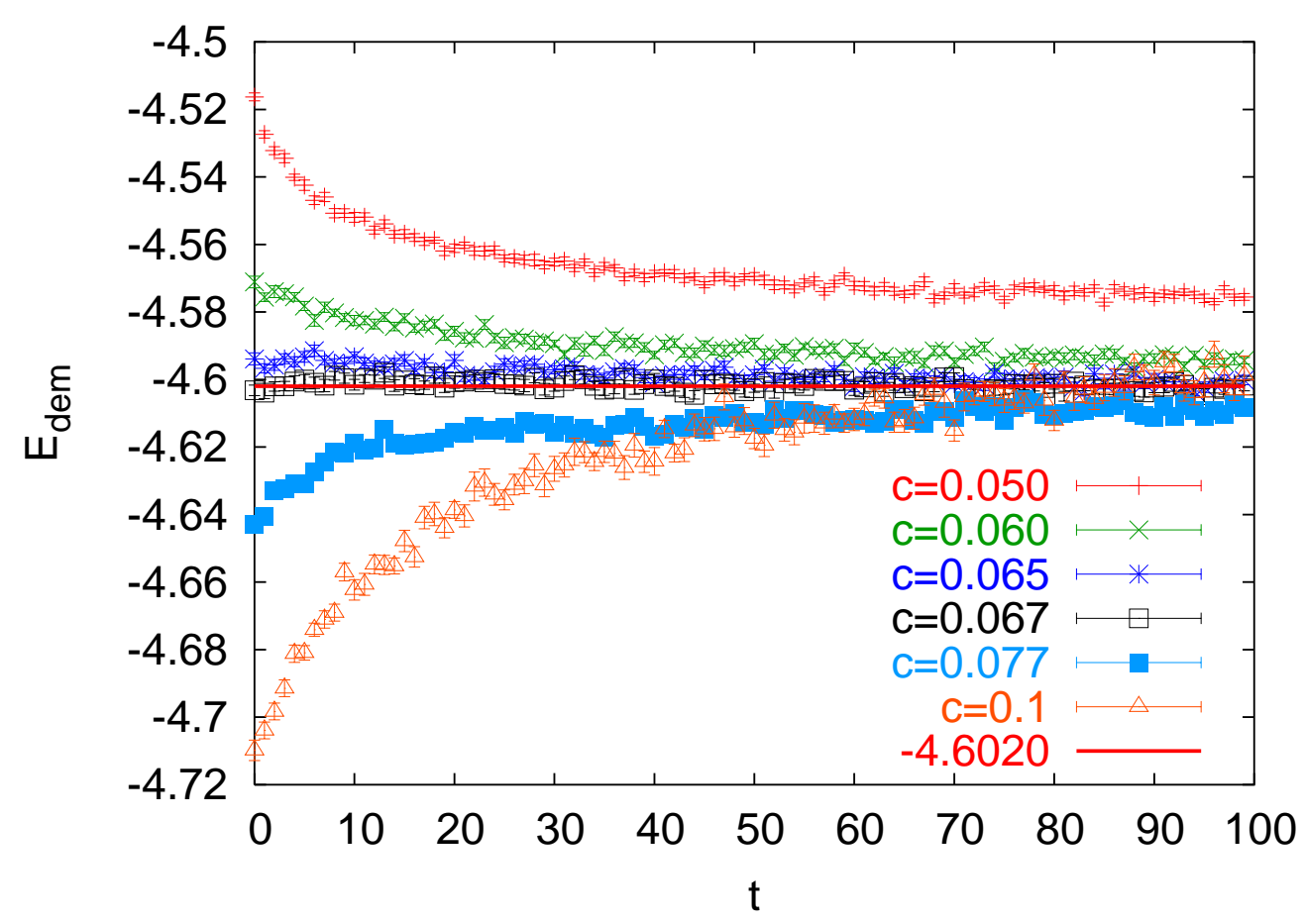

Figure 1: Demon fundamental representation energy flow for DSB decimation at various $c$ values.

numerical decimation procedure (so called "coarsening" effect). Letting

$$
\frac{a_{d e c}}{2 a}=\sqrt{\frac{\sigma_{d e c}}{\sigma_{0}}}, \quad \frac{a_{g e n}}{a_{d e c}}=\sqrt{\frac{\sigma_{g e n}}{\sigma_{d e c}}}, \quad \frac{a_{g e n}}{2 a}=\sqrt{\frac{\sigma_{g e n}}{\sigma_{0}}},
$$

the results are shown in Table 3. One now notes that coarsening is practically absent for values of

\begin{tabular}{|c||c|c|c|}
\hline $\mathrm{c}$ & $a_{\text {dec }} / 2 a$ & $a_{\text {gen }} / a_{\text {dec }}$ & $a_{\text {gen }} / 2 a$ \\
\hline 0.060 & $0.93(6)$ & $1.48(10)$ & $1.378(15)$ \\
0.065 & $0.95(5)$ & $1.16(7)$ & $1.109(20)$ \\
0.067 & $0.96(8)$ & $1.09(9)$ & $1.051(13)$ \\
0.068 & $0.97(2)$ & $0.99(3)$ & $0.966(15)$ \\
0.077 & $0.95(4)$ & $0.57(3)$ & $0.539(18)$ \\
\hline
\end{tabular}

Table 3: Lattice spacings ratios among original, decimated and effective-action-generated lattices.

$c$ close to those giving equilibrium values (no demon energy flow). For any other values, however, very substantial coarsening effects are generated (see Fig. 2). In other words, it is imperative to appropriately tune the decimation parameter to the effective action under consideration.

\subsection{Short distance and rotational invariance}

The Wilson action on a lattice of spacing $2 a$ would have a string tension $\sigma=(2 a / a)^{2} \sigma_{0}$. Therefore, we expect $\sigma \sim 0.1252$. This is very close to the string tension for the Wilson action at $\beta=2.31$, which is $\sigma=0.1230(14)$. We compare departures from rotational symmetry on 

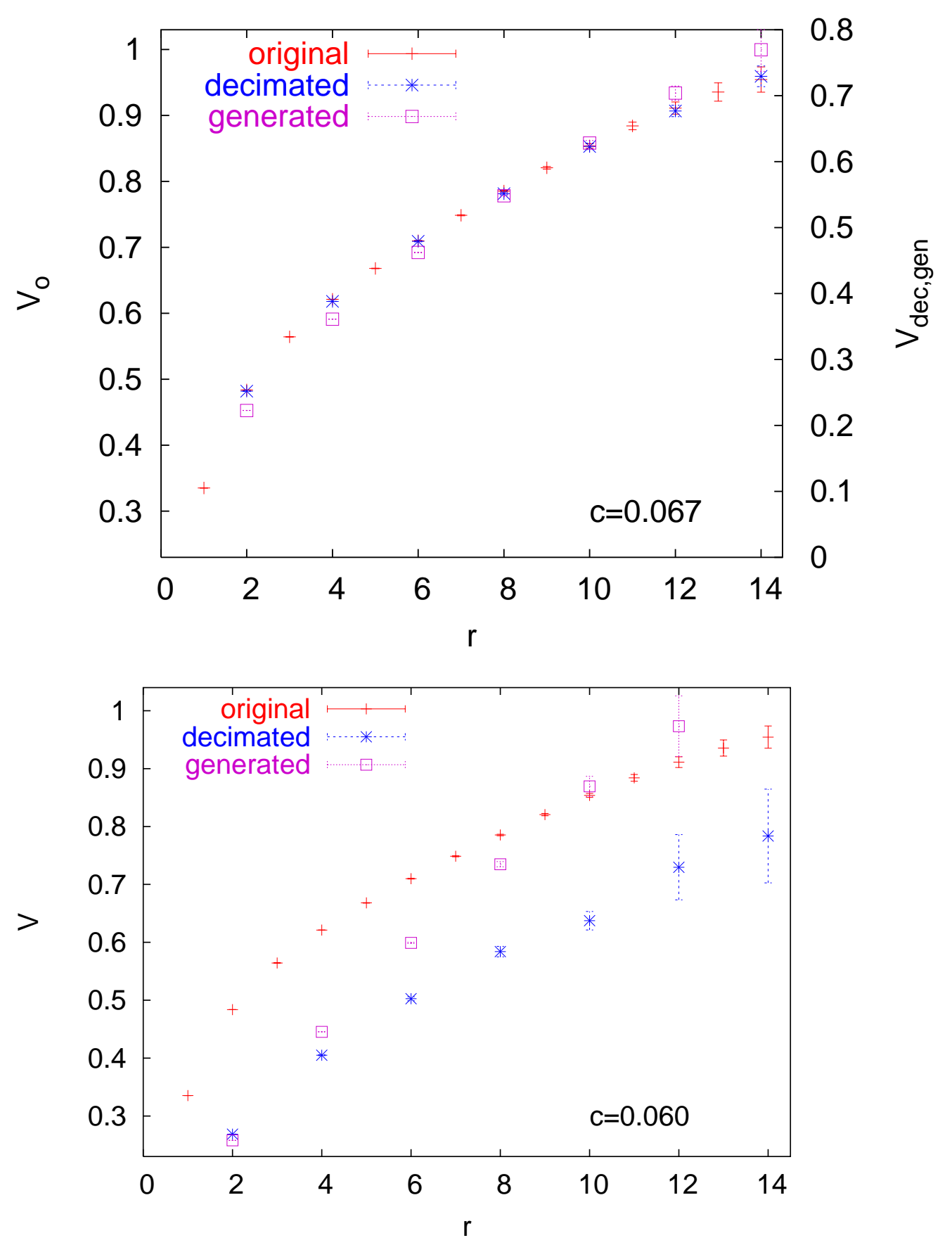

Figure 2: Static quark potential $V_{0}$ computed on original (undecimated), $V_{d e c}$ on DSB decimated ( $c=$ 0.067 ), and $V_{\text {gen }}$ on effective-action-generated lattices (top). The same, but for ( $c=0.060$ ) (bottom).

this Wilson action lattice and on the effective-action-generated lattice by looking at the off-axis correlators. We quantify the amount of rotational invariance violation by [4]:

$$
\delta_{V}^{2}=\sum_{o f f}\left(\frac{V_{o f f}(r)-V_{o n}(r)}{V_{o f f}(r) \delta V_{o f f}(r)}\right)^{2} / \sum_{o f f} \frac{1}{\delta V(r)^{2}}
$$


The results for first four off-axis points are: $\delta_{V}=0.045$ for Wilson, and $\delta_{V}=0.047$ for the effective action. They are comparable - there is no improvement at short distance.

\section{Extended action}

So far we worked with the multi-representation plaquette effective action (1.3). We saw that, appropriately tuning the decimation parameter $c$, this action reproduces long-distance behavior very well, but shows no particular improvement at short distances. In order to achieve such improvement (rotational invariance - removal of lattice artifacts) while preserving correct long distance behavior we next study a multi-representation action including the addition of the $1 \times 2$ loop (rectangle):

$$
S=\sum_{\text {plaq }} \sum_{j=1 / 2}^{j_{N}} \beta_{j}^{\text {plaq }}\left[1-\frac{1}{d_{j}} \chi_{j}\left(U_{p}\right)\right]+\sum_{\text {rect }} \sum_{j=1 / 2}^{j_{N}^{\prime}} \beta_{j}^{\text {rect }}\left[1-\frac{1}{d_{j}} \chi_{j}\left(U_{1 \times 2}\right)\right] .
$$

We report here some preliminary results with this extended action (3.1) in Table $\bigoplus$. The $c$ values

\begin{tabular}{|c|l|l|c|}
\hline$c$ & $\beta_{1 / 2}^{\text {plaq }}, \beta_{1}^{\text {plaq }}, \beta_{3 / 2}^{\text {plaq }}, \ldots$ & $\beta_{1 / 2}^{\text {rect }}, \beta_{1}^{\text {rect }}, \beta_{3 / 2}^{\text {rect }}, \ldots$ & $\sigma_{\text {gen }}$ \\
\hline \hline 0.90 & $3.4610(21),-0.0322(28)$, & $-0.3468(4), 0.0582(6)$, & $0.0384(34)[12]$ \\
& $0.0206(33),-0.0105(29)$ & $-0.0221(7), 0.0102(7)$ & \\
\hline 1.00 & $3.4039(19),-0.0357(28)$, & $-0.3262(4), 0.0564(6)$, & $0.0431(31)[22]$ \\
& $0.0187(35),-0.0112(32)$ & $-0.0209(6), 0.0097(5)$ & \\
\hline 1.40 & $3.1912(15),-0.0504(21)$, & $-0.2542(4), 0.0491(6)$, & $0.0322(21)[26]$ \\
& $0.0113(24),-0.0078(20)$ & $-0.0183(7), 0.0083(8)$ & \\
\hline 1.50 & $3.1518(21),-0.0576(22)$, & $-0.2398(4), 0.0466(4)$, & $0.0359(39)$ \\
& $0.0136(27),-0.0095(20)$ & $-0.0173(6), 0.0074(7)$ & \\
\hline
\end{tabular}

Table 4: The couplings of the extended effective action after Swendsen decimation starting from the Wilson action at $\beta=2.5$ and the string tension computed in the corresponding effective model. $c$ is the staple weight.

in Table $\bigoplus$ are all in the range that minimizes demon energies flow. We see that the resulting string tension is in the vicinity of the correct value. Coarsening effects range from $2 \%$ to $10 \%$. The main systematic error (not indicated) is due to error in $\beta$ determination. () stands for stochastic and [] for systematic (truncation) errors.

There is now significant restoration of rotational invariance (see Table 5 and Fig. 3) compared to the single plaquette action. The fluctuations in $\delta V$ values are primarily due to the use of the ansatz for $V_{o n}$.

\begin{tabular}{|c|c|}
\hline$c$ & $\delta V$ \\
\hline 0.80 & 0.0033 \\
0.90 & 0.0024 \\
1.00 & 0.0060 \\
1.50 & 0.0133 \\
\hline
\end{tabular}

Table 5: Amount of rotational invariance violation. The same decimations as in the previous table. 


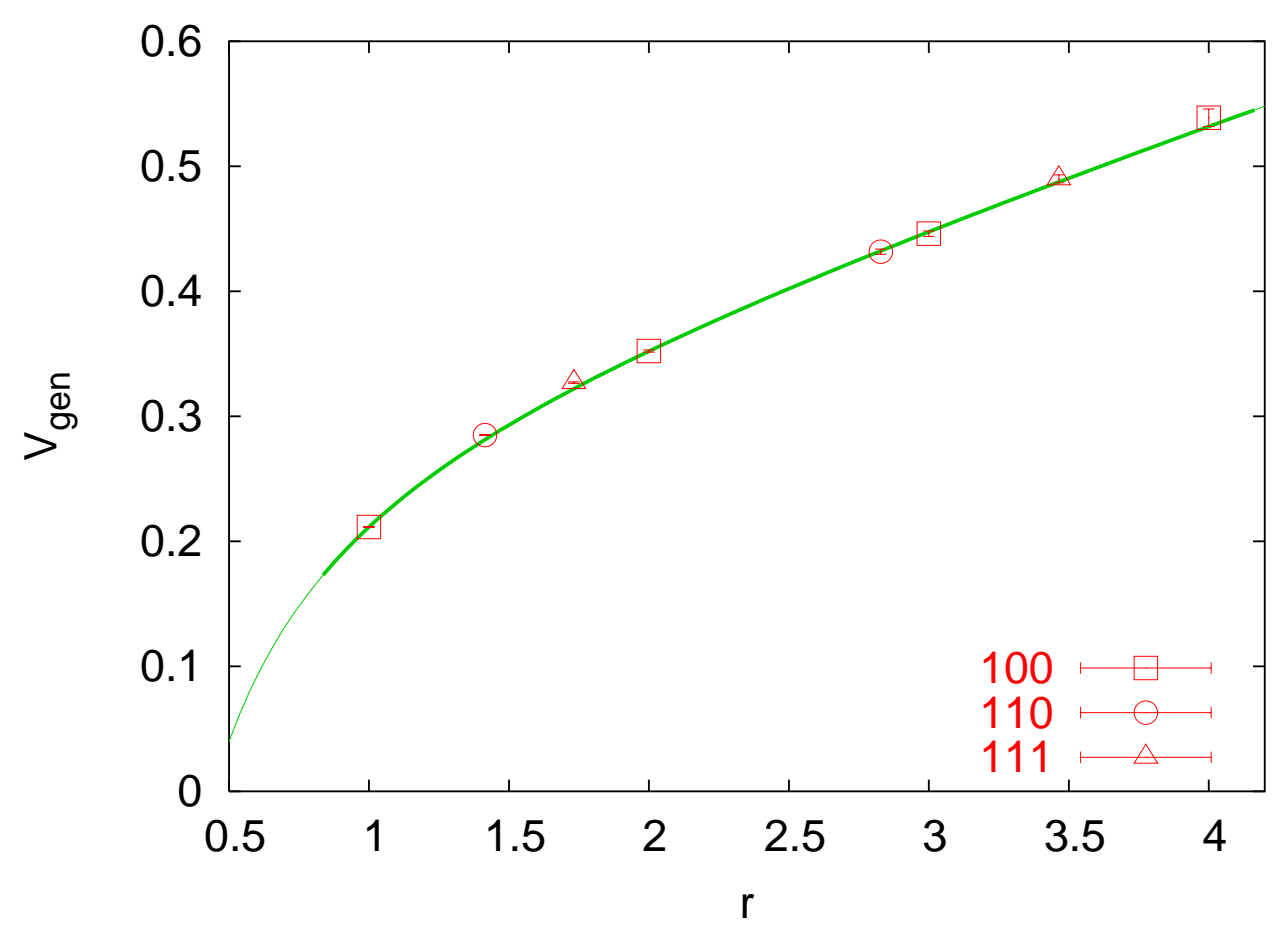

Figure 3: Static quark-antiquark potential on the effective-action-generated $c=1.50$ lattice; on and off axis separations.

In conclusion, our preliminary results indicate that, provided the decimation parameter(s) are tuned in the appropriate zone, the extended multi-representation (plaquette + rectangle)-action (3.1) does a good job of reproducing under decimation the physics of the original system over a wide scale regime.

\section{Acknowledgments}

We thank Academic Technology Services (UCLA) for computer support. This work was in part supported by NSF-PHY-0555693.

\section{References}

[1] E. T. Tomboulis and A. Velytsky, String tension and removal of lattice coarsening effects in Monte Carlo renormalization group, arXiv:0705.0383 [hep-lat].

[2] E. T. Tomboulis and A. Velytsky, Improving the improved action,' Phys. Rev. Lett. 98, 181601 (2007) [arXiv:hep-lat/0702027].

[3] E. T. Tomboulis and A. Velytsky, Renormalization group therapy, Phys. Rev. D 75, 076002 (2007) [arXiv:hep-lat/0702015].

[4] P. de Forcrand et al. [QCD-TARO Collaboration], Renormalization group flow of SU(3) lattice gauge theory: Numerical studies in a two coupling space, Nucl. Phys. B 577, 263 (2000)

[arXiv:hep-lat/9911033]. 